\title{
Metastasis of breast carcinoma to trigeminal nerve with numb chin syndrome: a case report and literature review
}

Ya Hui Lian

Jilin University First Hospital

De Zheng Kong

Jilin University First Hospital

Lin Jing Wang

Jilin University First Hospital

Chun Mei Wang

Jilin University First Hospital

Yang Yang Meng

Jilin University First Hospital

Hong Wei Zhou ( $\square$ doctorzhou74@163.com )

Jilin University First Hospital

\section{Case report}

Keywords: Breast carcinoma, trigeminal nerve, numb chin syndrome (NCS)

Posted Date: April 19th, 2019

DOl: https://doi.org/10.21203/rs.2.9241/v1

License: (1) This work is licensed under a Creative Commons Attribution 4.0 International License.

Read Full License 


\section{Abstract}

Background In general, common metastatic pathways for breast carcinoma include lung, liver, and bone. Described herein is a rare case of breast carcinoma metastasis to the trigeminal nerve. And numb chin syndrome(NCS) has a certain suggestive effect on the recurrence or progression of cancer patients. Case presentation a 54-year-old Chinese female was admitted to our hospital for progressive anaesthesia of the lower left lip and chin. She was diagnosed as breast ductal carcinoma 9 years ago. The brain MRI demonstrated dumbbell-shaped involvement of left trigeminal nerve which extended to left temporal lobe. Then we treated patient with radiotherapy, the patient's numbness relieved rapidly. Conclusion After review and analysis of the case, we found numb chin syndrome (NCS) is a "red flag" symptom of malignancy. 1 When cancer patients develop numb chin syndrome(NCS), we need to be alert to the possibility of distant metastasis. This case also suggests that clinicians should pay attention to the possibility of breast cancer metastasis to the trigeminal nerve.

\section{Background}

Common metastatic pathways for breast carcinoma include lung, liver, and bone. Direct trigeminal nerve involvement is more common in primary head and neck carcinoma and seldom reported in breast carcinoma. In this case, the patient with progressive anaesthesia of the lower left lip and chin as the main complaint, confirmed by imaging as left trigeminal nerve occupying lesion. We present this case to highlight the possibility of breast carcinoma metastasis to the trigeminal nerve, and need to improve the understanding of numb chin syndrome (NCS).

\section{Case Presentation}

A 54-year-old Chinese female was admitted to our department for progressive anaesthesia of the lower left lip and chin. She was diagnosed as hormone-receptor positive, human epidermal receptor-2 (HER-2) negative infiltrating breast ductal carcinoma 9 years ago. The initial treatment included radical mastectomy, adjuvant chemotherapy, radiotherapy and tamoxifen. In January, 2014, she developed bone metastasis and underwent ovarian castration and zoledronic acid. 16 months later, her bone metastases progressed, which prompted the switch to chemotherapy. In March, 2016, tumor markers increased and bone scan showed increased lesions. Therefore, she was enrolled in the clinical trial of chidamide. 10 months later (January, 2017), she developed liver metastases and progressive bone metastases. The subsequent brain magnetic resonance imaging (MRI) with contrast was normal (Figure 1). In April, 2017, she complained of numbness in left lip and chin without motor signs. Chemotherapy with Gemcitabine was given and in June, 2017 the patient developed lung metastases. 2 weeks before her referral to our department, she suffered from dizziness and progressive numbness. The cerebrospinal fluid analysis and skull base CT was normal. Her past history was unremarkable without any trauma and systemic diseases. The brain MRI demonstrated dumbbell-shaped involvement of left trigeminal nerve which extended to left temporal lobe measuring about $2.1 \times 2.0 \mathrm{~cm}$ (Figure 2 ). 
Radiotherapy was given and her numbness relieved rapidly.

\section{Discussion}

Numb chin syndrome (NCS), also called mental nerve neuropathy (MNN), is characterized by hypoesthesia, paresthesia or prickling pain over the chin, lower lip and gingiva mucosa in the distribution of the mental nerve or inferior alveolar nerve (IAN), and/or neuropathic weakness in the muscles of mastication. 2,3 The common cause of NCS is due to odontogenic infections, trauma, and tumors. Although NCS seems innocuous, more and more clinical cases show that NCS may be the primary clinical manifestation of metastasis in cancer patients. The mechanism included skull base metastases, neoplastic neural infiltration, leptomeningeal spread or jaw metastases. 4

NCS has been reported in many malignant diseases, including prostate cancer, colon cancer, lung cancer, breast cancer and so on. However, in patients with breast cancer, cases of NCS with trigeminal involvement are rarely reported. In our case, the patient had bone metastasis, liver metastasis, and lung metastasis in nine years. The patient occurred NCS before the lung metastasis, but at this time the brain MRI showed normal. After 5 months, the patient reported dizziness and progressive paralysis. At this time, the brain MRI clearly showed the space-occupying lesion of the left trigeminal nerve. After active radiation therapy, the patient's paralysis symptoms were quickly relieved.

In summary, NCS generally indicates a dismal prognosis with mean survival of 6.9 months and $79 \%$ mortality 4. This case is novel in that it reported a rare metastatic pathway in breast carcinoma and it illustrates the importance of awareness of NCS, so timely intervention might improve the patient's life quality and guarantee a better prognosis.

\section{Abbreviations}

NCS $₫$ Numb Chin Syndrome CT囚 Computed Tomography MRI囚Magnetic Resonance Imaging

\section{Declarations}

Ethics approval and consent to participate

Informed consent was obtained from the patient to publish her case $\$ and this study receive approval from the Research Ethics Committee of The First Hospital of Jilin University.

Consent for publication

Written informed consent for publication of this Case Report was obtained from the patient. A copy of written consent form is available for review to the Editor of this journal.

Availability of data and materials 
All data related to this case report are contained within the manuscript.

Competing interests

The authors declare that they have no competing interests.

Funding

The study was supported by special fund for health from financial department of Jilin province(grant number:3D516B843428)

Authors' contributions

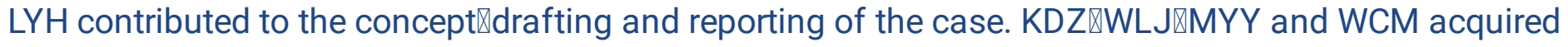
clinical data. ZHW contributed to revising the manuscript. All authors read and approved the final manuscript.

Acknowledgments

Not applicable.

\section{References}

1. Carbone M, Della Ferrera F, Carbone $L$ et al. Numb chin syndrome as first symptom of diffuse large Bcell lymphoma. Case Rep Dent 2014; 2014: 413162.

2. R.P.M. Bruyn, W. Boogerd The numb chin Clin Neurol Neurosurg, 93 (1991), pp. 187-193

3. Shin-YuLu,Shu-HuaHuang,Yen-HaoChen.Numb chin with mandibular pain or masticatory weakness as indicator for systemic malignancy - A case series study[J].Journal of the Formosan Medical Association,2017,116(11):897-906.

4. Brady B, Coughlan AM, O'Brien T. Numb Chin Syndrome: A Case Report. J Pain Symptom Manage 2016; 51: e3-4.

\section{Figures}




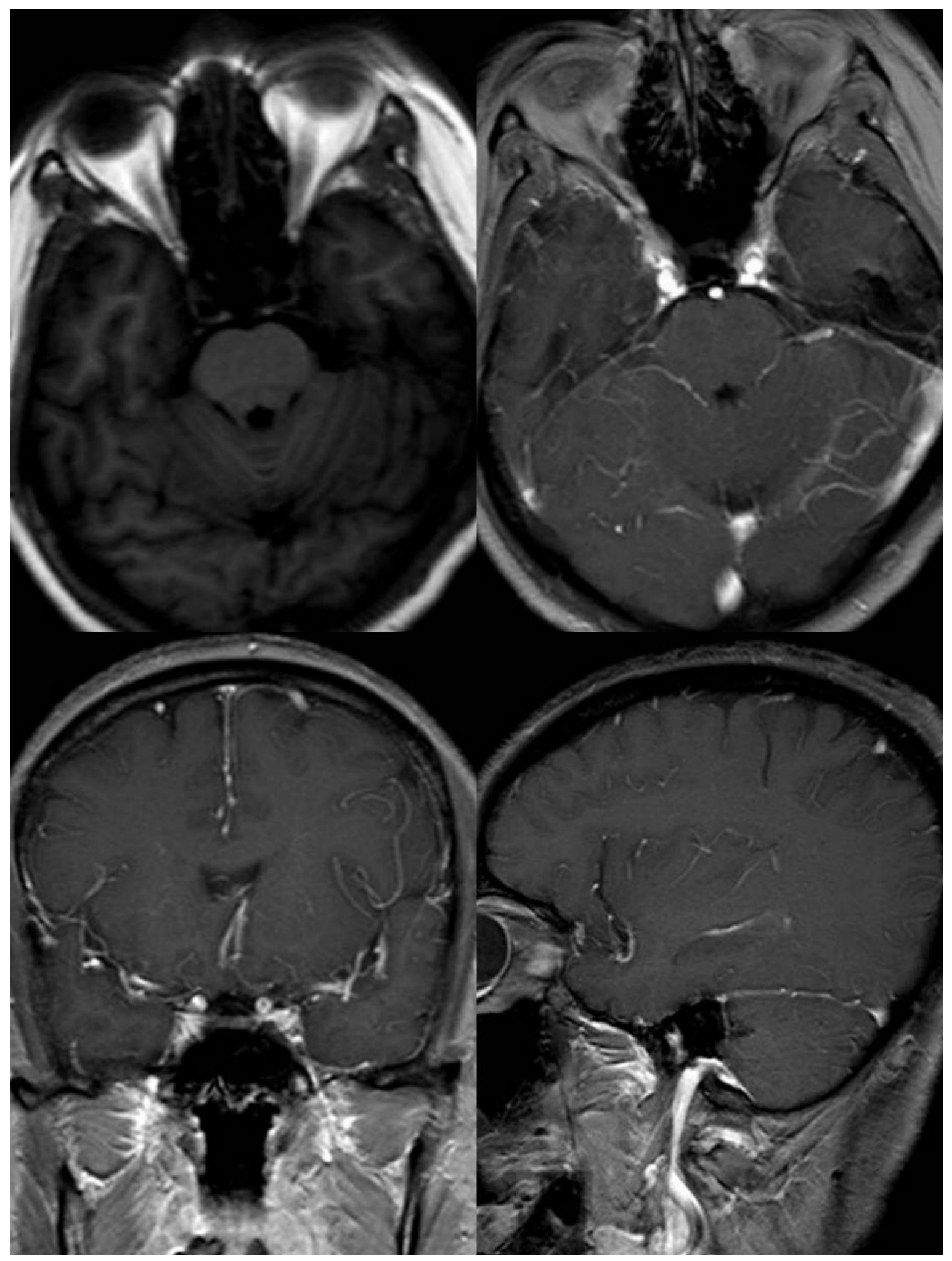

Figure 1

The patient's brain MRI in February 2017 showed no significant space-occupying lesions. 


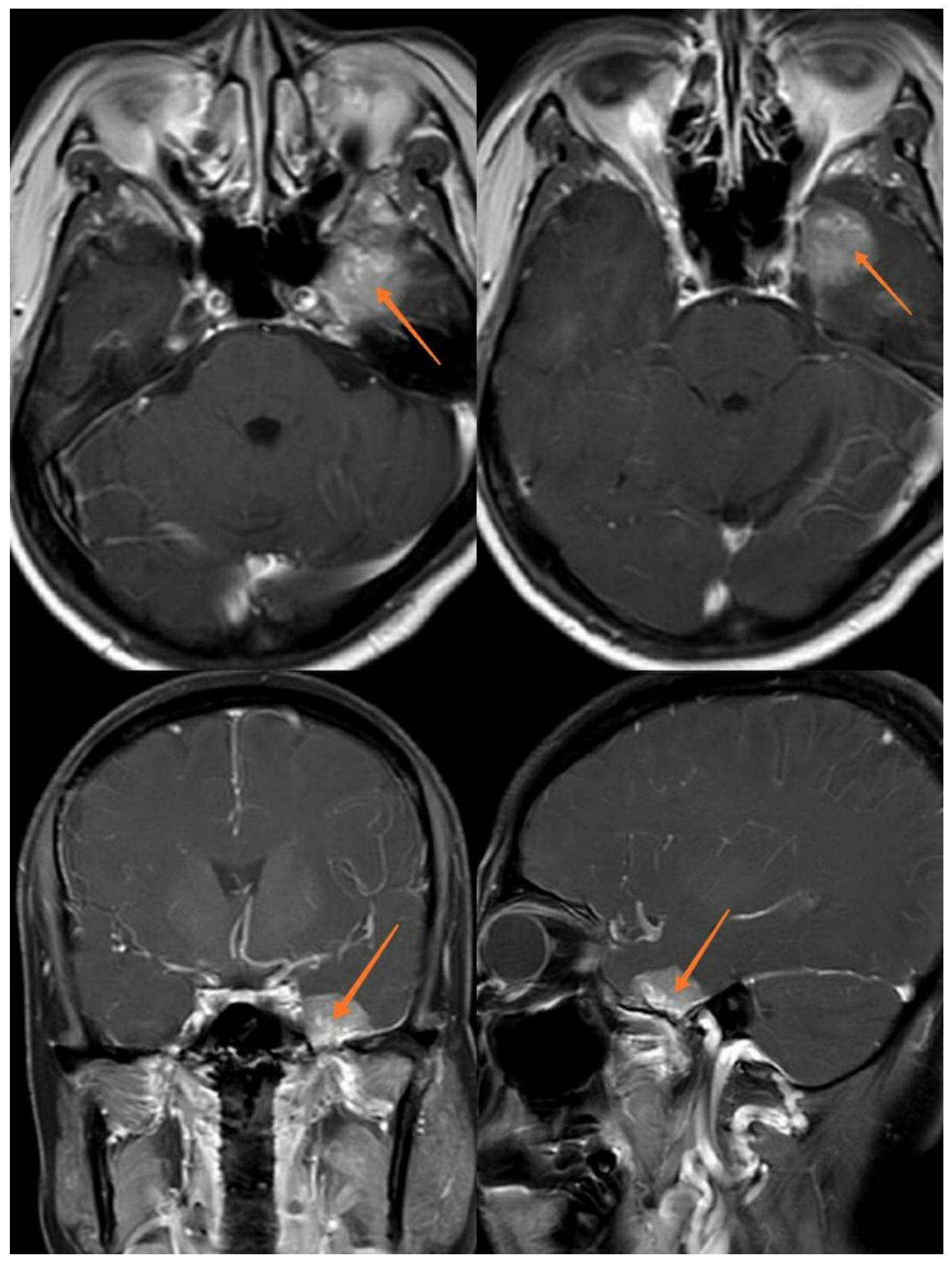

\section{Figure 2}

The patient's brain MRI in September 2017 showed the involvement of left trigeminal nerve which extended to left temporal lobe measuring about $2.1 \times 2.0 \mathrm{~cm}$ with dumbbell shape.

\section{Supplementary Files}

This is a list of supplementary files associated with this preprint. Click to download. 
- CAREchecklistEnglish20131.docx 